\title{
High-Yield Synthesis of Uniform Ag Nanowires with High Aspect Ratios by Introducing the Long-Chain PVP in an Improved Polyol Process
}

\author{
Jie-Jun Zhu, ${ }^{1,2}$ Cai-Xia Kan, ${ }^{3}$ Jian-Guo Wan, ${ }^{1}$ Min Han, ${ }^{1}$ and Guang-Hou Wang ${ }^{1}$ \\ ${ }^{1}$ National Laboratory of Solid State Microstructures and Department of Physics, Nanjing University, Nanjing 210093, China \\ ${ }^{2}$ Department of Applied Physics, Nanjing University of Technology, Nanjing 210009, China \\ ${ }^{3}$ College of Science, Nanjing University of Aeronautics and Astronautics, Nanjing 211100, China
}

Correspondence should be addressed to Guang-Hou Wang, ghwang@nju.edu.cn

Received 25 March 2011; Accepted 21 May 2011

Academic Editor: Wanqin Jin

Copyright () 2011 Jie-Jun Zhu et al. This is an open access article distributed under the Creative Commons Attribution License, which permits unrestricted use, distribution, and reproduction in any medium, provided the original work is properly cited.

Polyvinyl pyrrolidone (PVP) with different molecular weights was used as capping agent to synthesize silver nanowires through a polyol process. The results indicated that the yields and aspect ratios of silver nanowires were controlled by the chain length of PVP and increased with increasing the molecular weight (MW) of PVP. When the long-chain PVP-K90 $(\mathrm{MW}=800,000)$ was used, the product was uniform in size and was dominated by nanowires with high aspect ratios. The growth mechanism of the nanowires was studied. It is proposed that the chemical adsorption of $\mathrm{Ag}^{+}$on the PVP chains at the initial stage promotes the growth of $\mathrm{Ag}$ nanowires.

\section{Introduction}

Nanometer-sized noble metals are of great interest not only for their unique properties [1-3], but also for their potential applications in the fields of biomedical diagnostics [4], catalysis [5], optical devices [6], and data storage [7]. Among them, silver nanomaterials are particularly interesting due to their roles as substrates in studies of the surface-enhanced Raman scattering [8,9], nonlinear optical properties [10], and catalysis [11]. For the optical properties, absorption associated with the collective oscillation of the conduction electrons or surface plasmon resonance (SPR) has been studied for many decades. Both experimental and theoretical studies show that the number and position of plasmon resonances as well as the spectral range for the surface-enhanced Raman scattering (SERS) of metal nanostructures strongly correlate with their exact morphology and aspect ratios of the nanostructures $[12,13]$. In general, cylindrical Ag nanowires show one resonance, whereas several resonances are expected for Ag nanorods with triangle profiles [14]. For Ag nanoprisms, in-plane dipole SPR can be tuned across the entire visible spectrum from $\sim 400 \mathrm{~nm}$ to the near-infrared regions [15]. More recently, Ag nanowires have attracted much attention due to their important applications for fabricating nanoscale fluorescence sensors $[16,17]$, plasmonic fibers [18-21], and so on.

For fundamental and applied sciences, various methods have been adopted to prepare Ag nanostructures [22-25]. In recent years, Ag nanostructures have yielded a great deal of literature featuring on the anisotropic growth of crystals into defined morphologies, ranging from nanowires, nanorods, nanoplates, nanocubes, and branched multipods to highly faceted particles [13, 26-29]. Among these methods, the common chemical reduction method based on the polyol process, which is convenient, versatile, and low costing, is usually adopted for the synthesis of noble metal nanoparticles. By changing the preparation parameters in the system, all kinds of nanostructures with different morphologies can be obtained, and the morphology could be further well controlled through introducing capping agent. One good example is the synthesis of $\mathrm{Ag}$ nanowires using polyvinyl pyrrolidone (PVP) as structure-directing reagent developed by Xia and coworkers $[23,30]$. Based on the polyol method, large-scale uniform silver nanowires with high aspect ratios 
Table 1: Products obtained by using PVP with different MWs. $\left(T=150^{\circ} \mathrm{C}\right.$ and $\left.R=1\right)$. Note that $D$ and $L$ mean the average diameter and length of Ag nanowires, respectively.

\begin{tabular}{lcc}
\hline No & PVP & Final product (yields) and size of nanowires \\
\hline$(1)$ & K17 $(\mathrm{MW}=15000)$ & Nanoparticles $(\sim 60 \%)$ and nanowires $(\sim 40 \%) ; D: \sim 120 \mathrm{~nm}, L: \sim 3 \mu \mathrm{m}$ \\
$(2)$ & K25 $(\mathrm{MW}=38000)$ & Nanoparticles $(\sim 50 \%)$ and nanowires $(\sim 50 \%) ; D: \sim 100 \mathrm{~nm}, L: \sim 3 \mu \mathrm{m}$ \\
$(3)$ & K30 $(\mathrm{MW}=58000)$ & Nanoparticles $(\sim 10 \%)$ and nanowires $(90 \%) ; D: \sim 180 \mathrm{~nm}, L: \sim 8 \mu \mathrm{m}$ \\
$(4)$ & K60 $(\mathrm{MW}=200000)$ & Nanoparticles $(\sim 5 \%)$ and nanowires $(\sim 95 \%) ; D: \sim 120 \mathrm{~nm}, L: \sim 8 \mu \mathrm{m}$ \\
$(5)$ & K90 $(\mathrm{MW}=800000)$ & Nanowires $(\sim 99 \%) ; D: \sim 100 \mathrm{~nm}, L: \sim 10 \mu \mathrm{m}$ \\
\hline
\end{tabular}

were successfully achieved with [24] or without exotic seeds $[23,31]$.

Although the polyol process is a popular method of preparing metal nanostructures, so far most of the published works mainly focused on the synthesis process. In the case of the effect of capping agent PVP, although there are lots of documents studying the effect of the amount of PVP on determining the shape and size of Ag nanostructures, the investigations on the role of the molecular weight are still scarce and the effects on the synthesis of Ag nanostructures are not very clear until now [23, 24, 29].

In our previous work, we reported the preparation of $\mathrm{Ag}$ nanostructures with various shapes including nanoparticles, nanowires, nanorods, and nanocubes by the improved PVPmediated polyol process (PVP with $\mathrm{MW}=40000)[26,27]$. It was found that both the $\mathrm{PVP} / \mathrm{AgNO}_{3}$ ratio $R$ and reaction temperature $T$ played important roles and the favorable experimental parameters for synthesizing Ag nanowires were usually $R=5$ and $T=150^{\circ} \mathrm{C}$. If the $R$ value decreased to 1 , the perfect $\mathrm{Ag}$ nanocubes were the major products [27]. On the other hand, our recent inspiring investigation reveals that when the PVP with higher molecular weight was used, Ag nanowires with high yields and high aspect ratios were obtained at the condition of $R=1$ with the same temperature, indicating that the molecular weight of PVP is also a critical parameter for the synthesis of Ag nanowires.

In this paper, we successfully synthesized large-scale uniform silver nanowires with high aspect ratios by introducing the long-chain PVP $(\mathrm{MW}=800000)$ in an improved polyol process and investigated the effect of PVP molecular weight on the synthesis of Ag nanowires. The morphological evolution of the Ag nanostructures at various PVP molecular weights was studied in detail, and the growth mechanism of $\mathrm{Ag}$ nanowires is proposed linking both the structural characteristics of Ag nanostructures and the roles of PVP surfactants.

\section{Experimental}

$\mathrm{AgNO}_{3}$ ( $\geq 99.8 \%$, Sinopharm Chemical Reagent Co., Ltd.) and ethylene glycol (EG, Nanjing Chemical Reagent Co., Ltd.) were used in this work. PVP powders (Boai New Kaiyuan Pharmacy Co., Ltd.) with various average MW of 15000, 38000, 58000, 200000, and 800000 were chosen, which were marked as K17, K25, K30, K60, and K90, as shown in Table 1. All reagents were not purified further.
The improved PVP-mediated polyol process was used to synthesize the $\mathrm{Ag}$ nanowires. Firstly, $0.204 \mathrm{~g}$ of $\mathrm{AgNO}_{3}$ and $0.1332 \mathrm{~g}$ PVP were, respectively, solved in a $5 \mathrm{~mL}$ EG solvent. Then both solutions were simultaneously injected dropwise into $20 \mathrm{~mL}$ of EG solvent which was magnetically stirred at $150^{\circ} \mathrm{C}$ in a round-bottom flask. The injection time lasted about $3 \mathrm{~min}$. Magnetic stirring was applied throughout the entire synthesis. The molar ratio between the repeating unit of PVP and $\mathrm{AgNO}_{3}$ was fixed at $R=1$.

The reactant mixture changes quickly from colorless to yellow after the addition of a few drops of PVP and $\mathrm{AgNO}_{3}$ solutions. The mixtures turned from yellow to slightly opaque after $\sim 30 \mathrm{~min}$ and finally formed slightly green colloids for PVP-K17 and PVP-K25 after $~ 65$ min or gray white colloids for PVP-K30, PVP-K60, and PVP-K90 after $\sim 80 \mathrm{~min}$. For characterization, the mixture was sampled at different times and was then centrifuged repeatedly to remove possible contamination (EG and excess PVP).

For field-emission scanning electron microscopic (FESEM; Sirion200, FEI, Holland) and X-ray diffraction (XRD; Ultima-III; Rigaku, Tokyo, Japan) measurements, the condensed products were dispersed on the copper sheets and glass substrates, respectively. For transmission electron microscopy (TEM; JEM-2000EX; JEOL) and high-resolution (HR) TEM (Tecnai G2 F20, FEI) observations, the products were diluted with deionized water $(\sim \times 30)$, and a few drops of dispersions were placed onto the carbon-coated copper grids and dried at room temperature. Optical absorption spectra for the diluted samples were recorded on a UV-Vis-NIR spectrophotometer (U-3410, Hitachi). Fourier transformed infrared (FT-IR) spectra were obtained on a Bruker Vector 22 FT-IR spectrophotometer by using $\mathrm{KBr}$ pellets.

\section{Results and Discussion}

In our experiment, it was found that both yields and aspect ratios of the obtained $\mathrm{Ag}$ nanowires synthesized at $T=$ $150^{\circ} \mathrm{C}$ and $R=1$ increased with increasing the MW of PVP. Table 1 presents the yields and sizes of Ag nanowires with different PVP types. Figure 1 shows some typical FE-SEM results of samples no. $1-$ no. 5 in Table 1 . It can be seen that the Ag nanowires are not uniform in size and shape and a lot of irregular particles appear in the products when PVP-K17 and PVP-K25 were used (Figures 1(a) and 1(b)). For the case of sample-no. 3 (PVP-K30), the Ag nanowires (together with some cubic, tetrahedral, and other shaped nanoparticles) with high yields are not uniform with diameter ranging from 


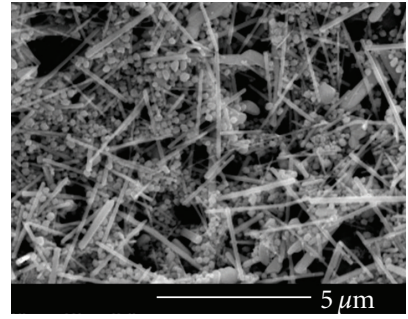

(a)

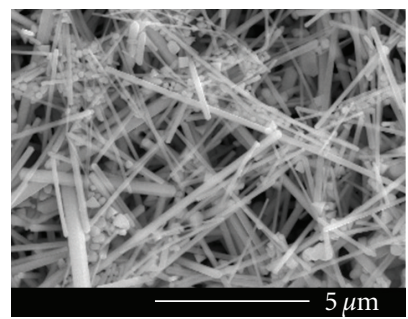

(c)

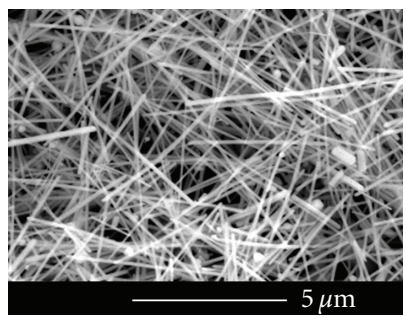

(e)

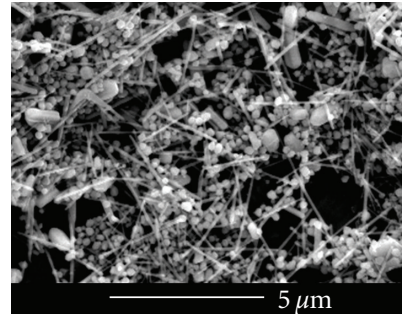

(b)

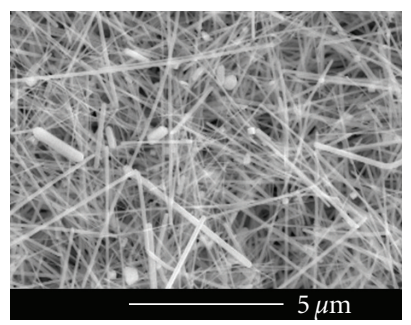

(d)

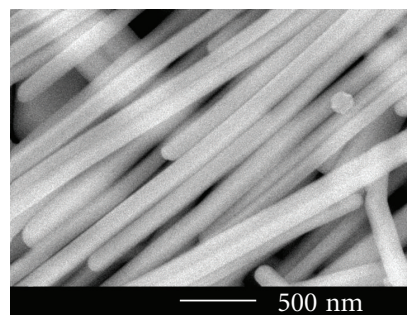

(f)
FIGURE 1: ((a)-(e)) FE-SEM images of the final products of sample no. 1-no. 5. (f) A magnified image of sample no. 5.

$40 \mathrm{~nm}$ to $380 \mathrm{~nm}$, as shown in Figure 1(c). With the MW of PVP increasing, the yields of the Ag nanowires increase further and almost no particles could be obsesrved in the product, as shown in Figures 1(d) and 1(e). Figure 1(f) is a magnified image of sample-no. 5 (PVP-K90); it is seen that the diameter of Ag nanowires is quite uniform and tends to self-assemble into two-dimensional arrays with solution evaporation during preparing sample.

Figure 2 presents the XRD pattern of sample no. 3-no. 5. The XRD pattern indicates that Ag nanowires synthesized using this solution-phase method are purely in the facecentred cube (fcc) phase. The lattice constant calculated from this XRD pattern was $4.092 \AA$, which is perfectly in agreement with the literature value of $4.086 \AA$. It is worth noting that the ratio of diffraction intensity between the (111) and (200) planes changed from 2.18 to 2.90 as PVP MW increased from 58000 to 800000 , possibly because of the higher yields of the Ag nanowires in the final products.

The TEM images (Figures 3(a), 3(b), and 3(c)) also indicate that the Ag nanowires of sample-no. 5 possess uniform diameter and high yield, just as what we observed in the SEM measurements. In order to obtain the detailed structural information of the Ag nanowires, we further performed the selected-area electron diffraction (SAED) pattern characterization and HRTEM observation, as shown in Figures 3(d)

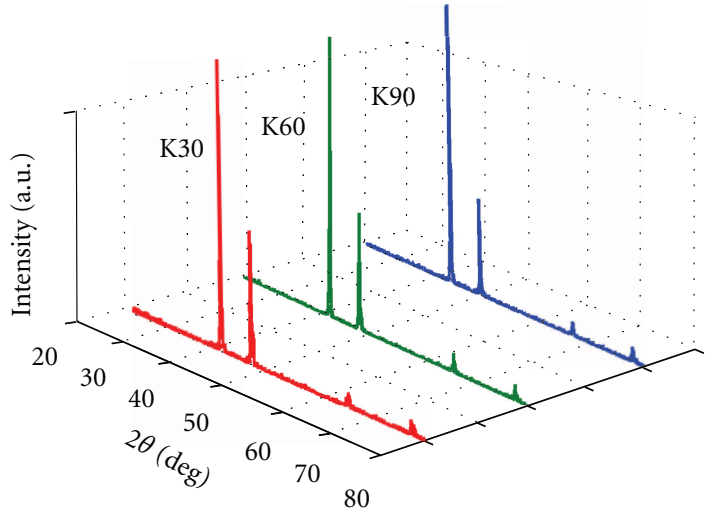

FIGURE 2: XRD patterns of the Ag nanowires synthesized using different MWs of PVP (K30, K60 and K90 with MW = 58000, 200000 and 800000$)$. The ratio of intensity between the (111) and (200) peaks was $2.18,2.4$ and 2.9 respectively.

and 3(e). The SAED pattern consists of a superimposition of contributions from different subunits. The spots from superimposition of [112] and [001] zone axes are indicated. According to the HRTEM image, the fringe spacing is about $0.145 \mathrm{~nm}$, which is in agreement with the (220) lattice spacing of Ag crystal, as shown in Figure 3(e). These results imply that the Ag nanowires should be fivefold symmetry rounding the long wire axis in the [110] direction, as widely reported about Ag nanowires [32-34].

Since Ag nanostructures with different shapes and sizes exhibit different SPR bands at different frequencies, we subsequently carried out UV-vis spectrum measurements for the samples. Figure 4 shows the optical extinction spectra of Ag colloid solutions synthesized using different MWs of PVP. One observes that all the products show the evident plasma peaks at $\sim 355 \mathrm{~nm}$ and $\sim 390 \mathrm{~nm}$, which should be attributed to the out-of-plane quadrupole resonance and out-of-plane dipole resonance of the Ag nanowires, respectively [35-37]. This observation indicates that all the synthesized products contain Ag nanowires. For the curves (a) and (b), there is another obvious peak at $\sim 445 \mathrm{~nm}$ ascribed to the SPR resonance of the $\mathrm{Ag}$ nanoparticles, and a shoulder peak around $\sim 445 \mathrm{~nm}$ also appears in curves (c) and (d), which indicates that the final products should be a mixture of Ag nanowires and a small amount of nanoparticles.

It is interesting that, with the MW of PVP increasing, the peak intensities at $\sim 355 \mathrm{~nm}$ and $\sim 390 \mathrm{~nm}$ increased while the peak intensity at $\sim 445 \mathrm{~nm}$ decreased. When the PVP molecular weight reaches 800000 , only the peak at $\sim 355 \mathrm{~nm}$ and $\sim 390 \mathrm{~nm}$ could be obsserved, indicating that there are almost only Ag nanowires in the final product. (see curve (e) for sample-no. 5). This is consistent with the results of SEM and TEM observations that Ag nanowires were more favorable products synthesized using higher MW of PVP. For the first time, to the best of our knowledge, uniform silver nanowires with high yields were successfully synthesized by using so-long-chain PVP $(\mathrm{MW}=800000)$ in a simple polyol process. Generally, PVP with MW $=40000$ or 55000 is used to prepare Ag nanowires. 


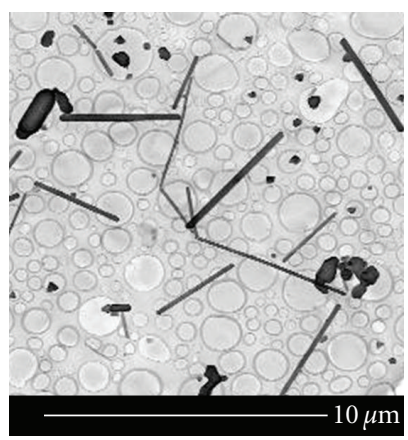

(a)

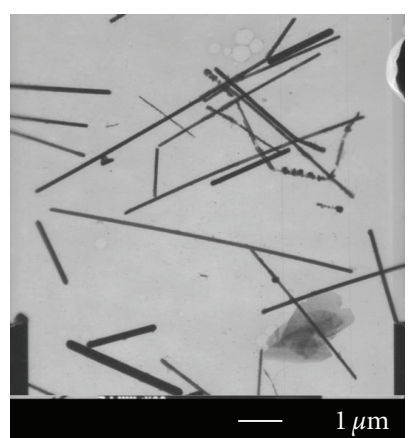

(c)

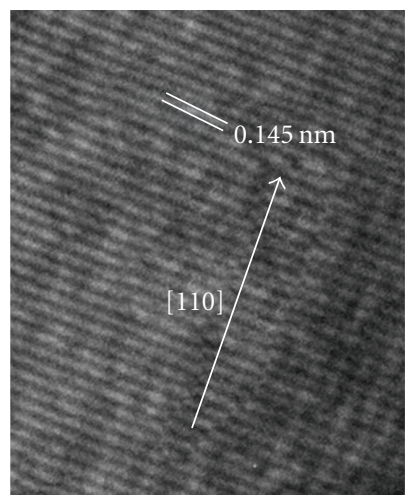

(e)

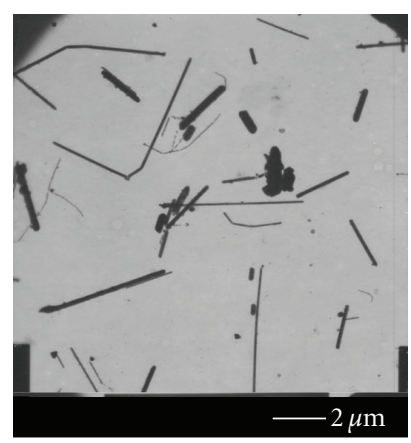

(b)

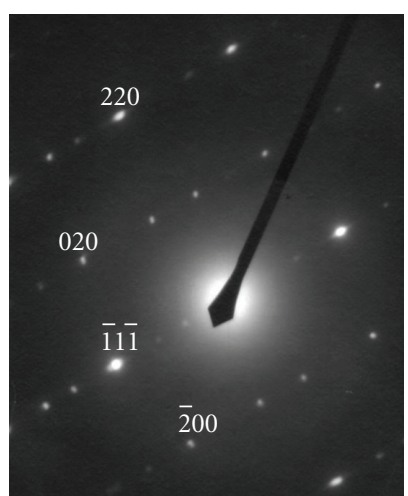

(d)

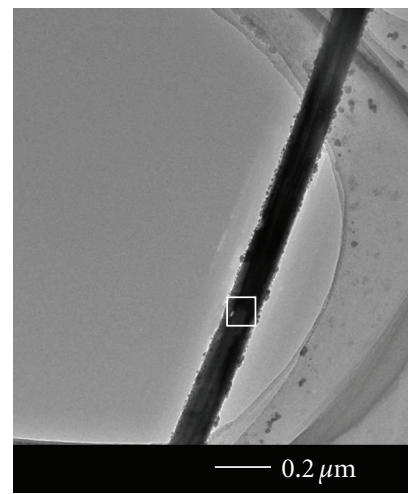

(f)
FIGURE 3: TEM images of the Ag nanoproducts using different MWs of PVP:(a) K30 (MW = 58000), (b) K60 (MW = 200000), and (c) K90 (MW = 800000). (d) A typical SAED of one Ag nanowire; the spots from superimposition of the [112] and [001] zone axis are indicated. (e) A typical HRTEM image of a selected area of an individual Ag nanowire. (f) The nanowire of sample no. 5 used for the SAED and HRTEM studies with the area marked with a white pane indicating where the HRTEM image was recorded.

According to all above results, we could conclude that the yields as well as the aspect ratios of the Ag nanowires increased with increasing the MW of PVP. In terms of the process of growth and reduction, EG is generally used as a reducing reagent while the PVP plays the role of surfactant during the preparation. This is true for the reduction of $\mathrm{Ag}^{+}$

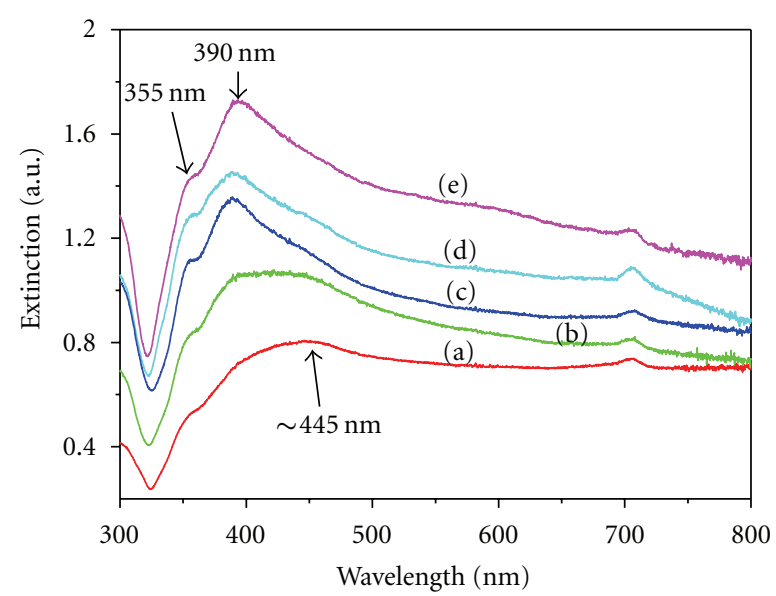
(a) PVP-K17
(d) PVP-K60
(b) PVP-K25
(e) PVP-K90
(c) PVP-K30

Figure 4: UV-visible extinction spectra of the final products synthesized using various MWs of PVP (a) K17 (MW = 15000), (b) K25 $(\mathrm{MW}=38000),(\mathrm{c}) \mathrm{K} 30(\mathrm{MW}=58000),(\mathrm{d}) \mathrm{K} 60(\mathrm{MW}=200000)$, and (e) K90 (MW = 800000). All solutions had been diluted by $\times 30$ with deionized water.

ions in hot EG solution, which was proved in our previous work [38], and the chemical reaction is as follows:

$$
\begin{gathered}
2 \mathrm{HOCH}_{2} \mathrm{CH}_{2} \mathrm{OH} \longrightarrow 2 \mathrm{CH}_{3} \mathrm{CHO}+2 \mathrm{H}_{2} \mathrm{O} \\
2 \mathrm{Ag}^{+}+2 \mathrm{CH}_{3} \mathrm{CHO} \longrightarrow \mathrm{CH}_{3} \mathrm{CO}-\mathrm{OCCH}_{3}+2 \mathrm{Ag}+2 \mathrm{H}^{+} .
\end{gathered}
$$

We now discuss the effect of PVP on the formation of Ag nanostructures. It is known that the growth morphology is often determined by the surface free energies under thermal equilibrium. On the nanometer scale, the transition fcc metals are favourable for the formation of fivefold twinned structure with their surfaces bounded by the lowest-energy $\left\{\begin{array}{lll}1 & 1 & 1\end{array}\right\}$ facets. However, crystal growth is usually far from the thermal equilibrium; thus, the shape is not characterized by minimizing the surface energy, but rather the growth rate of each face as determined by the kinetics [39]. In the study of the growth mechanism of anisotropic Ag nanostructures fabricated by the polyol process, a common attitude is that PVP interacts more strongly with Ag atoms on the $\{100\}$ facets than those on the $\{111\}$ facets, which act as a structure-directing agent as well as the capping agent preventing aggregation [30].

We know that PVP has the structure of polyvinyl skeleton with strong polar group (pyrrolidone ring), so it has an affinity toward many chemicals to form coordinative compounds. The polar groups, such as the $>\mathrm{C}=\mathrm{O}$ groups of $\mathrm{PVP}$ chain, can interact with metal ions and form coordinating complex (as seen in Scheme 1) [40]. In our previous work [41], Fourier transform infrared spectra of PVP and PVP-AgNO3 film were measured, and a shift in frequency of the $\mathrm{C}=\mathrm{O}$ in pyrrolidone ring indicates an interaction between the $\mathrm{AgNO}_{3}$ molecules and the PVP chains as in Scheme 1. These 


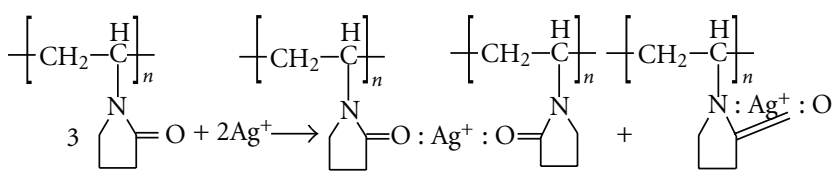

Scheme 1: Complexation of Ag ions with PVP chains.

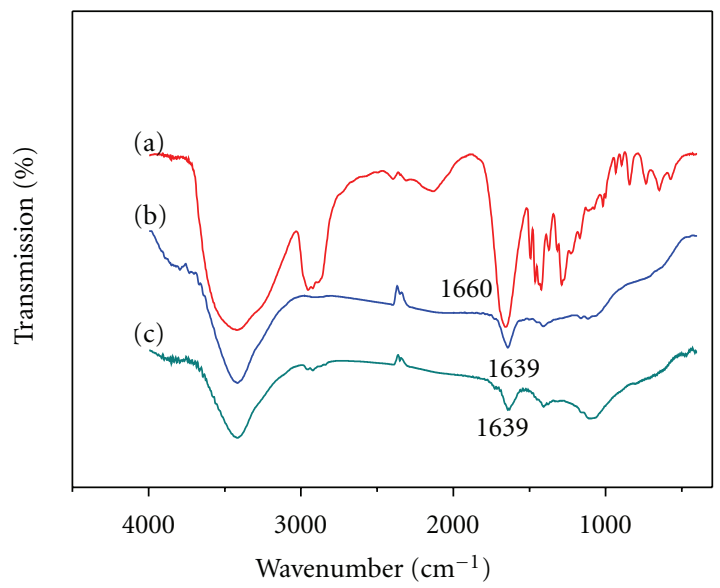

(a) PVP-K90

(b) PVP-Ag before washing

(c) PVP-Ag after washing

FIGURE 5: Infrared transmission spectra for PVP-K90 and the corresponding final products before and after washing.

reactions would create a state in which $\mathrm{Ag}^{+}$ions can be reduced to $\mathrm{Ag}^{0}$ to form extremely fine $\mathrm{Ag}$ embryos. Thus the PVP-Ag coordination complex compound would promote the formation of crystals once the mixture is dissolved in hot EG solution.

In our experiment, the chain length of PVP plays an important role in determining the yields and sizes of the silver nanowires. As for the long-chain PVP, which has higher degree of polymerization (DP) compared with the short one, the average number of repeating units in one PVP macromolecule chain $n$ is very high (from the PVP-K90 to PVP-K17, the average number $n$ is about 7207, 1802, 522, 342 , and 135, resp.). Thus, there are more carbonyl groups in one PVP macromolecule and more silver ions coordinated along the long chain of PVP. So it is suggested that the PVP with higher MW should more easily induce the PVPAg coordination compound to arrange in a one-dimensional (1D) manner and that linear Ag embryos with a longer size should be formed (as in Scheme 2). Accordingly, the embryos would continue to grow becoming silver nanowires, while the reduction reaction takes place in hot EG. As for the PVP with low MW, a relatively few silver ions coordinated along the short chain of PVP should result in the formation of silver nanorods with a shorter length, and those free silver ions which do not react with PVP would be attributed to the formation of $\mathrm{Ag}$ nanoparticles. So the previous adsorption of $\mathrm{Ag}^{+}$on the PVP chains in the initial stage and the chain length of PVP both are critical factors to synthesis of $1 \mathrm{D}$ silver nanostructures. We suggest that the coordination compound

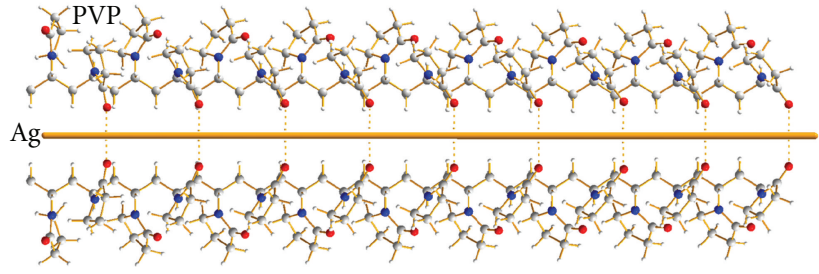

Scheme 2: Schematic diagram of 1D structure for PVP-Ag coordination compound. The red, blue, and white spheres represent the atoms of oxygen, nitrogen, and carbon, respectively.

of $\mathrm{Ag}^{+}$and the PVP with high MW should facilitate the formation of Ag nanowires with high aspect ratios.

Furthermore, to explore the surface chemical state of the final Ag nanowires, we also investigated the infrared spectra of pure PVP and the final product before and after washing. Figure 5 presents the infrared transmission spectrum for PVP-K90 and the corresponding final product which shows that the peak of the $\mathrm{C}=\mathrm{O}$ at $1660 \mathrm{~cm}^{-1}$ was slightly widened and shifted to approximately $1639 \mathrm{~cm}^{-1}$ after coordination with silver. The results suggest that the bonding between PVP and the metal crystal remains even when the metal ions are reduced. The fact that there still exists the $\mathrm{C}=\mathrm{O}$ peak at $1639 \mathrm{~cm}^{-1}$ in the spectra of the product after washing verified a certain chemical action between PVP and silver. This result is accordant to investigations of some other groups. For example, Xie et al. suggest that one monolayer of the PVP molecules might be adsorbed on the surface of the Ag nanowires through Ag-O coordination according to the results of thermogravimetric analysis (TGA), X-ray photoelectron spectroscopy (XPS), and Fourier transform Raman spectra (FT-Raman) [42]. The Ag nanowires modified by long-chain PVP would be helpful for self-assembly into ordered two-dimensional arrays for the PVP layer providing possible interaction among the neighboring Ag nanowires.

\section{Conclusions}

We have successfully synthesized uniform Ag nanowires with high yields by using a simple and improved PVP-mediated polyol process through only adjusting the MW of PVP at $150^{\circ} \mathrm{C}$ and $R=1$. We found the chain length of PVP played a critical role in directing the $1 \mathrm{D}$ growth of $\mathrm{Ag}$ nanostructures. With the MW of PVP increasing from 15000 to 800000 , the yields of Ag nanowires improved from $40 \%$ to $99 \%$ and the average length increased from $3 \mu \mathrm{m}$ to $10 \mu \mathrm{m}$; moreover, the uniformity of size became better. The growth mechanism of silver nanowires proposes that the chemical adsorption of $\mathrm{Ag}^{+}$on the PVP chains promotes a linear arrangement of the reduced Ag atoms and that more Ag atoms or small particles should be attributed to the growth of Ag nanowires naturally. High-yield synthesis of prefect silver nanowires with high aspect ratios requires only the long-chain PVP usage and need not introduce any exotic seeds and ions. The method is very simple and economical and promotes the potential applications of silver nanowires in lots of areas such as SERS, electronic, photonic, and optoelectronic devices. 


\section{Acknowledgments}

This work was supported by the National Natural Science Foundation of China (Grant no. 90606002), the National Key Projects for Basic Research of China (Grant no. 2010CB923401), and the Program for New Century Excellent Talents in University of China (Grant no. NCET-07-0422). The authors thank Dr. Zhaosheng Li for his great help with the XRD measurements in his research group.

\section{References}

[1] Z. L. Wang, T. S. Ahmad, and M. A. El-Sayed, "Steps, ledges and kinks on the surfaces of platinum nanoparticles of different shapes," Surface Science, vol. 380, no. 2-3, pp. 302-310, 1997.

[2] E. Hao, S. Li, R. C. Bailey, S. Zou, G. C. Schatz, and J. T. Hupp, "Optical properties of metal nanoshells," Journal of Physical Chemistry B, vol. 108, no. 4, pp. 1224-1229, 2004.

[3] J. Beermann, S. I. Bozhevolnyi, and V. Coello, "Modeling of nonlinear microscopy of localized field enhancements in random metal nanostructures," Physical Review B, vol. 73, no. 11, Article ID 115408, 10 pages, 2006.

[4] X. H. Huang, P. K. Jain, I. H. El-Sayed, and M. A. El-Sayed, "Gold nanoparticles: interesting optical properties and recent applications in cancer diagnostics and therapy," Nanomedicine, vol. 2, no. 5, pp. 681-693, 2007.

[5] Y. Nishihata, J. Mizuki, T. Akao et al., "Self-regeneration of a Pd-perovskite catalyst for automotive emissions control," Nature, vol. 418, no. 6894, pp. 164-167, 2002.

[6] S. A. Maier, M. L. Brongersma, P. G. Kik, S. Meltzer, A. A. G. Requicha, and H. A. Atwater, "Plasmonics-a route to nanoscale optical devices," Advanced Materials, vol. 13, no. 19, pp. 1501-1505, 2001.

[7] L. A. Peyser, A. E. Vinson, A. P. Bartko, and R. M. Dickson, "Photoactivated fluorescence from individual silver nanoclusters," Science, vol. 291, no. 5501, pp. 103-106, 2001.

[8] M. L. Coluccio, G. Das, F. Mecarini et al., "Silver-based surface enhanced Raman scattering (SERS) substrate fabrication using nanolithography and site selective electroless deposition," Microelectronic Engineering, vol. 86, no. 4-6, pp. 10851088, 2009.

[9] J. M. McLellan, Z. Y. Li, A. R. Siekkinen, and Y. Xia, "The SERS activity of a supported ag nanocube strongly depends on its orientation relative to laser polarization," Nano Letters, vol. 7, no. 4, pp. 1013-1017, 2007.

[10] Q. Q. Wang, J. B. Han, H. M. Gong et al., "Linear and nonlinear optical properties of ag nanowire polarizing glass," Advanced Functional Materials, vol. 16, no. 18, pp. 2405-2408, 2006.

[11] S. S. Hassani, M. R. Ghasemi, M. Rashidzadeh, and Z. Sobat, "Nano-sized silver crystals and their dispersion over $\alpha$ alumina for ethylene epoxidation," Crystal Research and Technology, vol. 44, no. 9, pp. 948-952, 2009.

[12] I. O. Sosa, C. Noguez, and R. G. Barrera, "Optical properties of metal nanoparticles with arbitrary shapes," Journal of Physical Chemistry B, vol. 107, no. 26, pp. 6269-6275, 2003.

[13] B. Wiley, Y. Sun, B. Mayers, and Y. Xia, "Shape-controlled synthesis of metal nanostructures: the case of silver," Chemistry, vol. 11, no. 2, pp. 454-463, 2005.

[14] P. J. Kottman, O. J. F. Martin, D. R. Smith, and S. Schultz, "Plasmon resonances of silver nanowires with a nonregular cross section," Physical Review B, vol. 64, no. 23, Article ID 235402, 10 pages, 2001.

[15] R. Jin, Y. C. Cao, E. Hao, G. S. Métraux, G. C. Schatz, and C. A. Mirkin, "Controlling anisotropic nanoparticle growth through plasmon excitation," Nature, vol. 425, no. 6957, pp. 487-490, 2003.

[16] T. Shegai, Y. Huang, H. Xu, and M. Käll, "Coloring fluorescence emission with silver nanowires," Applied Physics Letters, vol. 96, no. 10, Article ID 103114, 3 pages, 2010.

[17] K. Aslan, Z. Leonenko, J. R. Lakowicz, and C. D. Geddes, "Fast and slow deposition of silver nanorods on planar surfaces: application to metal-enhanced fluorescence," Journal of Physical Chemistry B, vol. 109, no. 8, pp. 3157-3162, 2005.

[18] H. Ditlbacher, A. Hohenau, D. Wagner et al., "Silver nanowires as surface plasmon resonators," Physical Review Letters, vol. 95, no. 25, Article ID 257403, 4 pages, 2005.

[19] A. W. Sanders, D. A. Routenberg, B. J. Wiley, Y. Xia, E. R. Dufresne, and M. A. Reed, "Observation of plasmon propagation, redirection, and fan-out in silver nanowires," Nano Letters, vol. 6, no. 8, pp. 1822-1826, 2006.

[20] M. W. Knight, N. K. Grady, R. Bardhan, F. Hao, P. Nordlander, and N. J. Halas, "Nanoparticle-mediated coupling of light into a nanowire," Nano Letters, vol. 7, no. 8, pp. 2346-2350, 2007.

[21] Z. Y. Fang, Y. W. Lu, L. R. Fan, C. F. Lin, and X. Zhu, "Surface plasmon polariton enhancement in silver nanowirenanoantenna structure," Plasmonics, vol. 5, no. 1, pp. 57-62, 2010.

[22] B. R. Martin, D. J. Dermody, B. D. Reiss et al., "Orthogonal self-assembly on colloidal gold-platinum nanorods," Advanced Materials, vol. 11, no. 12, pp. 1021-1025, 1999.

[23] Y. Sun and Y. Xia, "Large-scale synthesis of uniform silver nanowires through a soft, self-seeding, polyol process," Advanced Materials, vol. 14, no. 11, pp. 833-837, 2002.

[24] M. Tsuji, Y. Nishizawa, K. Matsumoto, M. Kubokawa, N. Miyamae, and T. Tsuji, "Effects of chain length of polyvinylpyrrolidone for the synthesis of silver nanostructures by a microwavepolyol method," Materials Letters, vol. 60, no. 6, pp. 834-838, 2006.

[25] R. Becker, F. Söderlind, B. Liedberg, and P. O. Käll, "Synthesis of silver nanowires in aqueous solutions," Materials Letters, vol. 64, no. 8, pp. 956-958, 2010.

[26] C.-X. Kan, J.-J. Zhu, and X.-G. Zhu, "Silver nanostructures with well-controlled shapes: synthesis, characterization and growth mechanisms," Journal of Physics D, vol. 41, no. 15, Article ID 155304, 9 pages, 2008.

[27] J. J. Zhu, C. X. Kan, X. G. Zhu et al., "Synthesis of perfect silver nanocubes by a simple polyol process," Journal of Materials Research, vol. 22, no. 6, pp. 1479-1485, 2007.

[28] Y. Sun, B. Mayers, and Y. Xia, "Transformation of silver nanospheres into nanobelts and triangular nanoplates through a thermal process," Nano Letters, vol. 3, no. 5, pp. 675679, 2003.

[29] X. Liu, F. Zhang, R. Huang, C. Pan, and J. Zhu, "Capping modes in PVP-directed silver nanocrystal growth: multitwinned nanorods versus single-crystalline nano-hexapods," Crystal Growth and Design, vol. 8, no. 6, pp. 1916-1923, 2008.

[30] Y. Sun, B. Mayers, T. Herricks, and Y. Xia, "Polyol synthesis of uniform silver nanowires: a plausible growth mechanism and the supporting evidence," Nano Letters, vol. 3, no. 7, pp. 955960, 2003.

[31] K. K. Caswell, C. M. Bender, and C. J. Murphy, "Seedless, surfactantless wet chemical synthesis of silver nanowires," Nano Letters, vol. 3, no. 5, pp. 667-669, 2003. 
[32] H. Hofmeister, S. A. Nepijko, D. N. Ievlev, W. Schulze, and G. Ertl, "Composition and lattice structure of fivefold twinned nanorods of silver," Journal of Crystal Growth, vol. 234, no. 4, pp. 773-781, 2002.

[33] H. Chen, Y. Gao, H. Yu et al., "Structural properties of silver nanorods with fivefold symmetry," Micron, vol. 35, no. 6, pp. 469-474, 2004.

[34] C. Ni, P. A. Hassan, and E. W. Kaler, "Structural characteristics and growth of pentagonal silver nanorods prepared by a surfactant method," Langmuir, vol. 21, no. 8, pp. 3334-3337, 2005.

[35] J. P. Kottman, O. J. F. Martin, D. R. Smith, and S. Schultz, "Plasmon resonances of silver nanowires with a nonregular cross section," Physical Review B, vol. 64, no. 23, Article ID 235402, 10 pages, 2001.

[36] J. P. Kottmann, O. J. F. Martin, D. R. Smith, and S. Schultz, "Spectral response of plasmon resonant nanoparticles with a non-regular shape," Optics Express, vol. 6, no. 11, pp. 213-219, 2000.

[37] S. Chen and D. L. Carroll, "Synthesis and characterization of truncated triangular silver nanoplates," Nano Letters, vol. 2, no. 9, pp. 1003-1007, 2002.

[38] C. X. Kan, C. S. Wang, J. J. Zhu, and H. C. Li, "Formation of gold and silver nanostructures within polyvinylpyrollidone (PVP) gel," Journal of Solid State Chemistry, vol. 183, no. 4, pp. 858-865, 2010.

[39] Z. L. Wang, "Transmission electron microscopy of shapecontrolled nanocrystals and their assemblies," Journal of Physical Chemistry B, vol. 104, no. 6, pp. 1153-1175, 2000.

[40] P. Jiang, S. Y. Li, S. S. Xie, Y. Gao, and L. Song, "Machinable long PVP-stabilized silver nanowires," Chemistry, vol. 10, no. 19, pp. 4817-4821, 2004.

[41] C. X. Kan, W. P. Cai, C. C. Li, and L. D. Zhang, "Optical studies of polyvinylpyrrolidone reduction effect on free and complex metal ions," Journal of Materials Research, vol. 20, no. 2, pp. 320-324, 2005.

[42] Y. Gao, P. Jiang, D. F. Liu et al., "Evidence for the monolayer assembly of poly(vinylpyrrolidone) on the surfaces of silver nanowires," Journal of Physical Chemistry B, vol. 108, no. 34, pp. 12877-12881, 2004. 

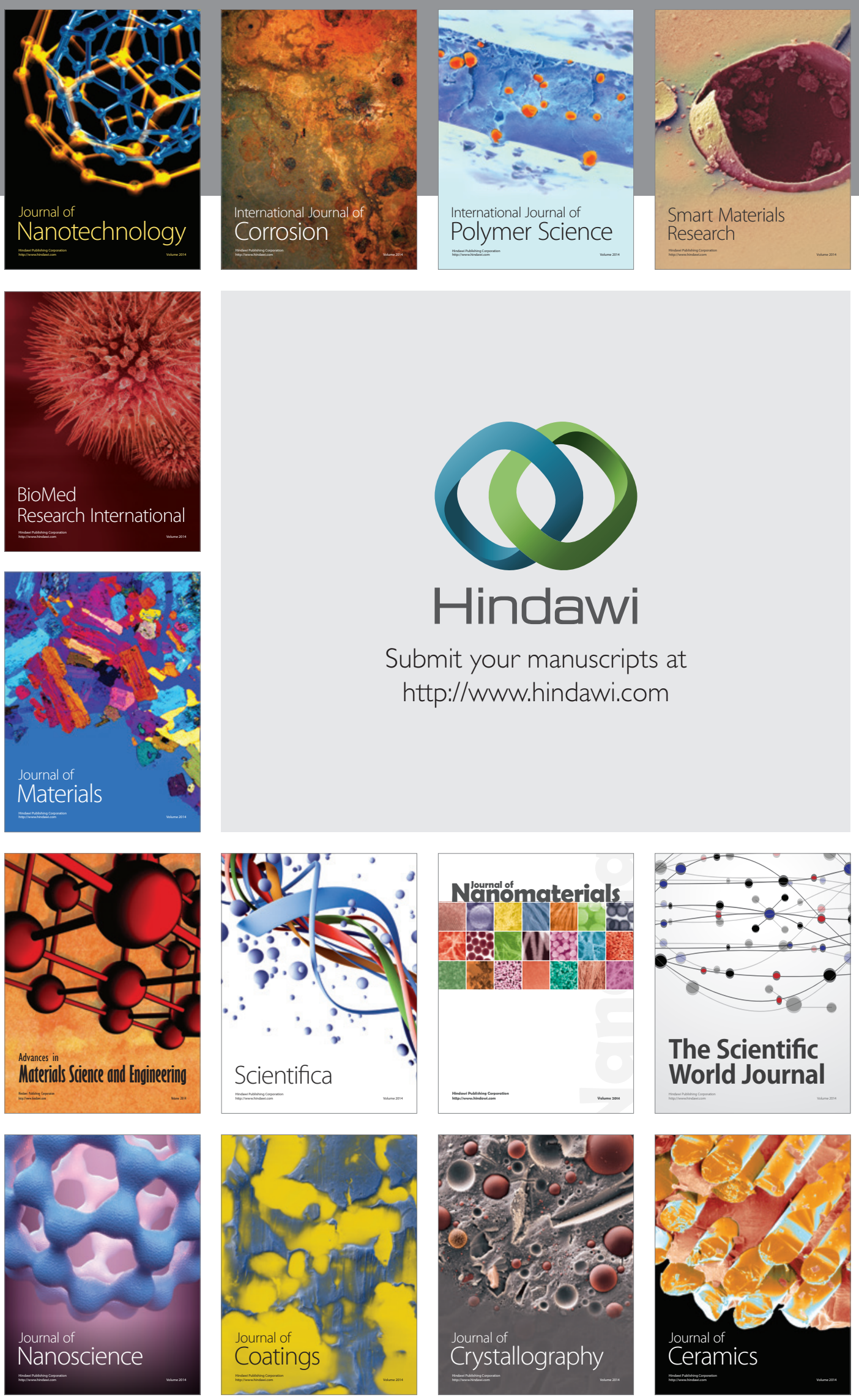

The Scientific World Journal

Submit your manuscripts at

http://www.hindawi.com

\section{World Journal}

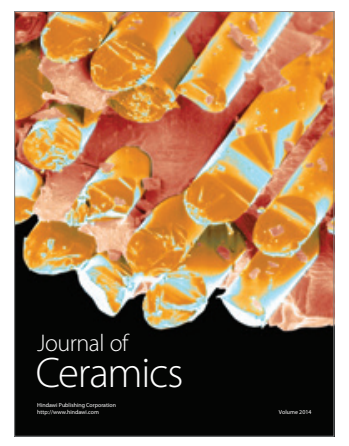

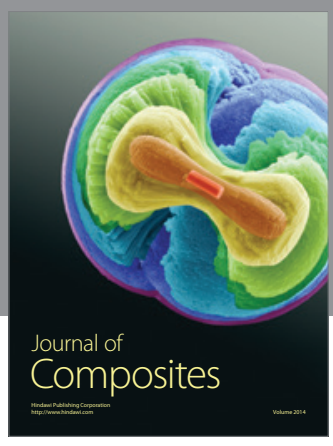
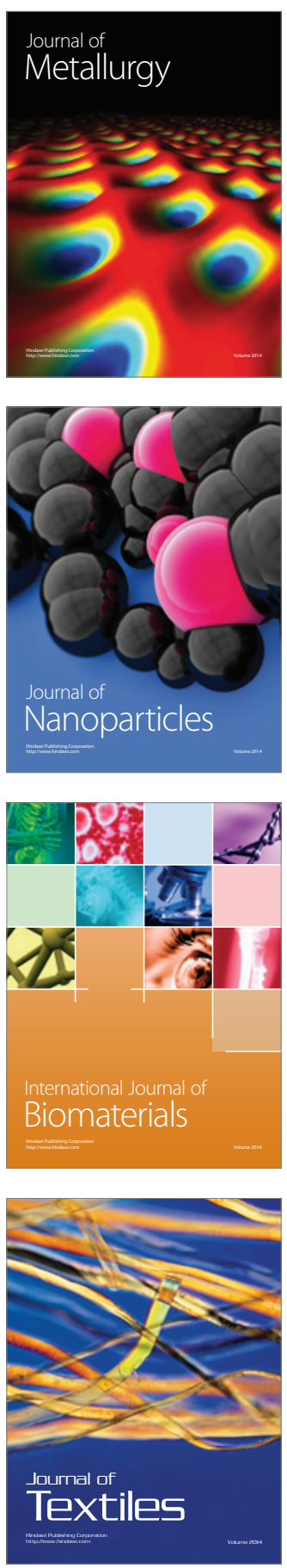